\title{
DYNAMIC PERFORMANCE OF A BACK-TO-BACK HVDC STATION BASED ON VOLTAGE SOURCE CONVERTERS
}

\author{
Mohamed Khatir — Sid-Ahmed Zidi \\ Samir Hadjeri — Mohammed-Karim Fellah *
}

\begin{abstract}
The recent developments in semiconductors and control equipment have made the voltage source converter based high voltage direct current (VSC-HVDC) feasible. This new DC transmission is known as "HVDC Light or "HVDC Plus by leading vendors. Due to the use of VSC technology and pulse width modulation (PWM) the VSC-HVDC has a number of potential advantages as compared with classic HVDC. In this paper, the scenario of back-to-back VSC-HVDC link connecting two adjacent asynchronous AC networks is studied. Control strategy is implemented and its dynamic performances during disturbances are investigated in MATLAB/Simulink program. The simulation results have shown good performance of the proposed system under balanced and unbalanced fault conditions.
\end{abstract}

K e y w or d s: HVDC, voltage source converter (VSC), IGBT, SPWM, control design

\section{NOMENCLATURE}

$U_{L} \quad$ the sinusoidal AC voltage in the AC network

$U_{V(1)} \quad$ the fundamental line to line voltage (valve side)

$X_{L} \quad$ the leakage reactance of the transformer

$\delta \quad$ phase shift between $U_{L}$ and $U_{V}(1)$

$I_{V} \quad$ source current

$L, R \quad$ phase reactor inductance and resistance

$C \quad$ DC side capacitance

$\omega \quad$ source voltage angular frequency

$m \quad$ modulation index

$P, Q \quad$ AC active, reactive power inputs

$U_{d}, I_{d}, P_{d}$ DC side voltage, current, power

$\alpha, \beta \quad$ stationary $\alpha-\beta$ axis

$d, q \quad$ synchronous $d-q$ axis

$p, n \quad$ positive, negative components

* reference value for controller

\section{INTRODUCTION}

The availability of the modern semiconductor devices such as the Insulated Gate Bipolar Transistor (IGBT) [1] has led to the development of a new generation of power electric converters. These devices, unlike the conventional thyristors which have no intrinsic turn-off ability, are of the fully controlled type. The most common converters, which employ the self commutating, high voltage, high current, and high switching frequency power electronic devices, are the Voltage Source Converters (VSC). A number of FACTS controllers which use VSC as their basic building block have been already in operation in various parts of the world. The most popular controllers are: the STATCOM, the SSSC, the UPFC, and the voltage source converter based HVDC transmission (VSCHVDC) $[2,3]$.

The VSC-HVDC system is the most recent HVDC technology. It consists of two VSC, one of which operates as a rectifier and the other as an inverter. The two converters are connected either back-to-back or joined by a DC cable, depending on the application. Its main function is to transmit a constant DC power from the rectifier station to the inverter station, with high controllability. The VSC-HVDC has several main advantages against the conventional HVDC based on thyristors. They are $[4,5]$ :

1. Independent control of the active and reactive power output from each terminal.

2. Reduced requirements for harmonic filters.

3. Improvements of the power quality and system stability.

4. Elimination of the requirement for a local power generation.

These features make the VSC-HVDC attractive for connection of weak AC system, island networks, and renewable energy sources, which may be located in remote area, to a main grid.

This paper presents the elements of back-to-back VSCHVDC where the converter stations are located at the same site and transmission line or cable is not needed. The paper wills first give a brief description about the VSC-HVDC transmission system and its terminal control functions. Following that, typical operating contingency scenarios are simulated in order to evaluate transient performance. The simulation results confirm that the control strategy has fast response and strong stability.

* Electrical Engineering Department Intelligent Control \& Electrical Power Systems Laboratory (ICEPS) Djillali Liabes University, Sidi Bel-Abbes, 22000, Algeria, med_khatir@yahoo.fr, sbzidi@yahoo.fr, shadjeri2@yahoo.fr, mkfellah@yahoo.fr 


\section{PRINCIPLE OF VSC OPERATION}

The fundamentals of VSC transmission operation may be explained by considering each terminal as a voltage source connected to the AC transmission network via a three-phase reactor. The two terminals are interconnected either back-to-back or through a DC link, as schematically shown in Fig. 1.

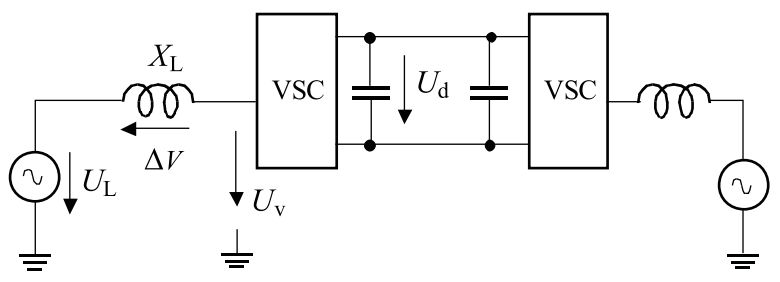

Fig. 1. Basic VSC transmission

The VSC converter can be represented as a variable $\mathrm{AC}$ voltage source where the amplitude, the phase and the frequency can be controlled independently of each other. It means that the VSC bridge can be seen as a very fast controllable synchronous machine with the instantaneous phase voltage $\left(U_{V}\right.$ in Fig. 1$)$, as described by the following equation

$$
U_{V}=\frac{1}{2} U_{d} m \sin (\omega t+\delta)+\text { harmonic_terms } .
$$

Variables $m$ and $\delta$ can be adjusted independently by the VSC controller to give any combination of voltage magnitude and phase shift in relation to the fundamental frequency voltage in the $\mathrm{AC}$ system. As a result, the voltage drop ( $\Delta V$ shown in Fig. 1) across the reactor ( $X_{L}$ shown in Fig. 1) can be varied to control the active and reactive power flows.

The active power flow between the converter and the network can be controlled by changing the phase angle $(\delta)$ between the fundamental frequency voltage generated by the converter $\left(U_{V(1)}\right)$ and the voltage $\left(U_{L}\right)$ on the bus. The power is calculated according to equation (2) assuming a lossless reactor $\left(X_{L}\right)$.

$$
P=\frac{U_{L} U_{V(1)}}{X_{L}} \sin \delta
$$

The reactive power flow is determined by the amplitude of $U_{V(1)}$ which is controlled by the width of the pulses from the converter bridge. The reactive power is calculated according to equation (3). The maximum fundamental voltage out from the converter depends on the DC voltage.

$$
Q=\frac{U_{L}\left(U_{L}-U_{V(1)} \cos \delta\right)}{X_{L}}
$$

\section{SYSTEM UNDER STUDY}

A $200 \mathrm{MW}( \pm 100 \mathrm{kV}$ DC) asynchronous interconnection is used to transmit power from a $230 \mathrm{kV}, 2000 \mathrm{MVA}$, $60 \mathrm{~Hz}$ network having a SCR of 10 to $230 \mathrm{kV}, 1000 \mathrm{MVA}$, $50 \mathrm{~Hz}$ network having a SCR of 5 . As shown in Fig. 2, it consists of DC capacitors, two converters, passive highpass filters, phase reactors, and transformers.

\subsection{The AC systems}

The AC networks, both at the rectifier and inverter end, are modeled as infinite sources separated from their respective commutating buses by system impedances. The impedances are represented as $\mathrm{L}-\mathrm{R} / \mathrm{L}$ networks having the same damping at the fundamental and the third harmonic frequencies. The impedance angles of the receiving end and the sending end systems are selected to be 80 degrees. This is likely to be more representative in the case of resonance at low frequencies [6].

\subsection{The Converters}

The converters are VSC employing IGBT power semiconductors, one operating as a rectifier and the other as an inverter. The rectifier and the inverter are threelevel Neutral Point Clamped (NPC) VSC converters using close IGBT/Diodes. The two converters are connected either back-to-back connection (no DC cable).

\subsection{The transformers and phase reactors}

A 200 MVA converter transformer $\left(Y_{g} / \Delta\right)$ is used to permit the optimal voltage transformation. The $0.15 \mathrm{pu}$ phase reactor with the $0.15 \mathrm{pu}$ transformer leakage reactance permits the VSC output voltage to shift in phase and amplitude with respect to the AC system Point of Common Coupling (PCC) and allows control of converter active and reactive power output. The tap position is rather at a fixed position determined by a multiplication factor applied to the primary nominal voltage of the converter transformers. The multiplication factors are chosen to have a modulation index around 0.85 (transformer ratios of 0.915 on the rectifier side and 1.015 on the inverter side).

\subsection{AC filters}

To meet AC system harmonic specifications, AC filters form an essential part of the scheme. They can be connected as shunt elements on the AC system side or the converter side of the converter transformer. Since there are only high frequency harmonics, shunt filtering is therefore relatively small compared to the converter rating. The 40 Mvar shunt AC filters are 27th and 54th high-pass tuned around the two dominating harmonics.

\subsection{DC capacitors}

On the DC side there are two capacitor stacks of the same size $(70 \mu \mathrm{F})$. The objective for the DC capacitor is primarily to provide a low inductive path for the turnedoff current and energy storage to be able to control the 


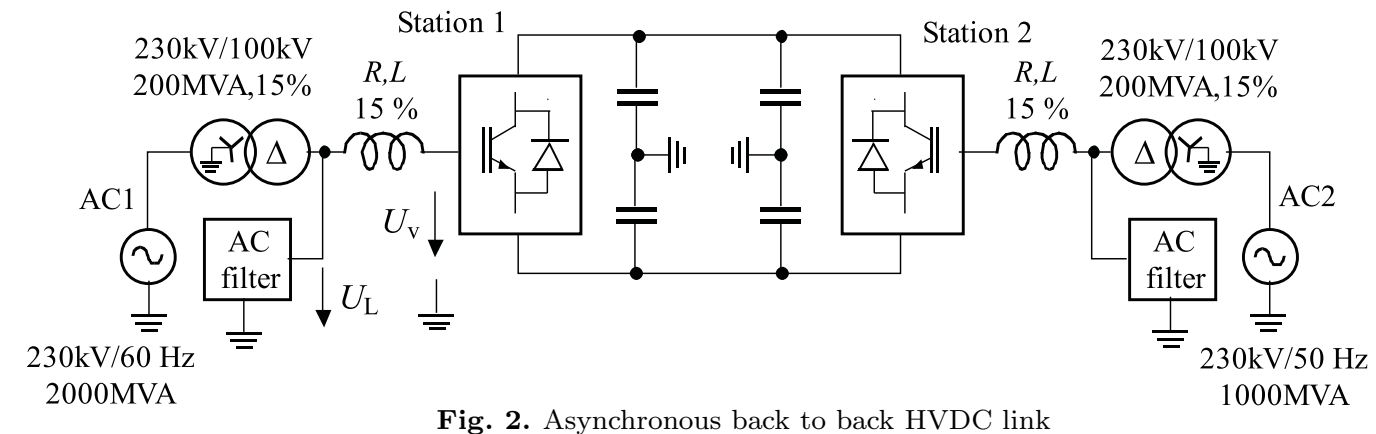

Fig. 2. Asynchronous back to back HVDC link

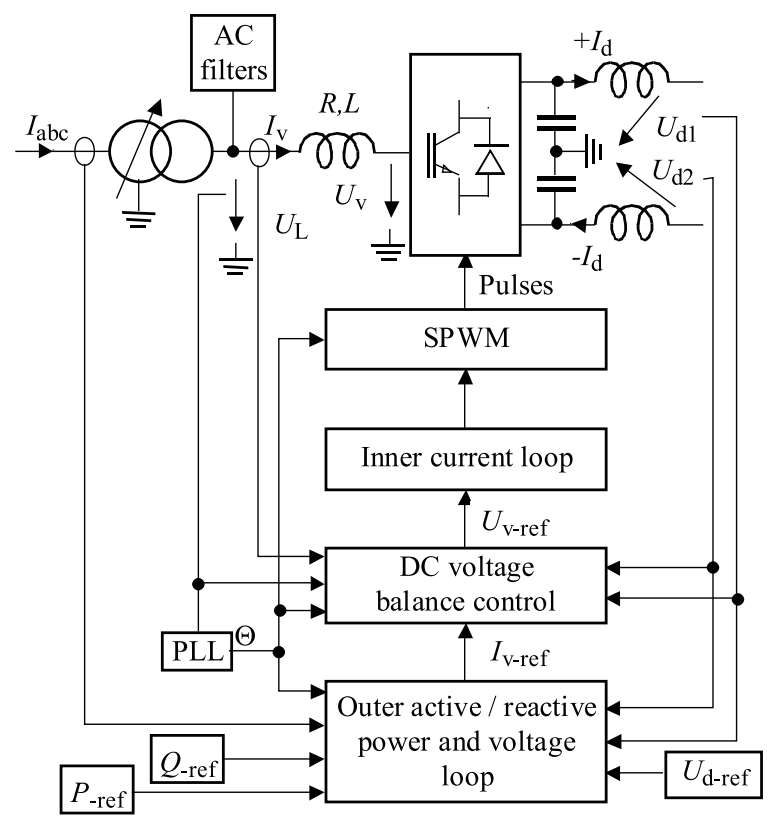

Fig. 3. Asynchronous back to back HVDC link

power flow. The capacitor also reduces the voltage ripple on the DC side.

\section{CONTROL STRATEGY}

For the convenience of the analysis, we have the following assumption: the station 1 controls both the active and reactive power exchanges between the $\mathrm{AC}$ system and $\mathrm{DC}$ link. The station 2 has the DC voltage and the reactive power as its control goals [7]. Figure 3 shows an overview diagram of the VSC control system and its interface with the main circuit. The converter 1 and converter 2 controller designs are identical. The two controllers are independent with no communication between them.

\subsection{Phase locked loop}

The phase locked loop (PLL) is used to synchronise the converter control with the line voltage and also to compute the transformation angle used in the $d-q$ transformation. The PLL block measures the system frequency and provides the phase synchronous angle $\Theta$ for the $d-q$ transformations block. In steady state, $\sin (\Theta)$ is in phase with the fundamental (positive sequence) of the $\alpha$ component and phase A of the point of common coupling voltage $\left(U_{a b c}\right)$.

\subsection{Outer active and reactive power and DC voltage loop}

The active power (at station 1) or the DC voltage (at station 2) is controlled by the control of $\delta$ and the reactive power is controlled by the control of the modulation index $(m)$. The instantaneous active and reactive power of the inverter on the valve side can be expressed in terms of the $d q$ component of the current and the voltage on the valve side as follows

$$
\begin{aligned}
& p=\frac{3}{2} \operatorname{Re}\left(\bar{u}_{L}^{d q} \bar{i}_{v}^{* d q}\right)=\frac{3}{2}\left(u_{L d} i_{v d}+u_{L q} i_{v q}\right), \\
& p=\frac{3}{2} \operatorname{Im}\left(\bar{u}_{L}^{d q} \bar{i}_{v}^{* d q}\right)=\frac{3}{2}\left(-u_{L d} i_{v q}+u_{L q} i_{v d}\right) .
\end{aligned}
$$

If the reference of the $d q$-frame is selected such that the quadrature component of the voltage is being very small and negligible $\left(u_{L q} \approx 0\right)$ then the equations (4) and (5) indicate that the active and the reactive power are proportional to the $d$ and $q$ component of the current respectively. Accordingly, it is possible to control the active power (or the DC voltage) and the reactive power (or the AC bus voltage) by control of the current components $i_{v d}$ and $i_{v q}$ respectively. The active and reactive power and voltage loop (shown in Fig. 4) contains the outer loop regulators that calculate the reference value of the converter current vector $\left(I_{d q}^{*}\right)$ which is the input to the inner current loop.

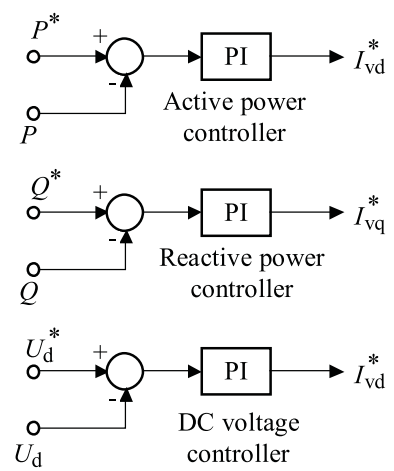

Fig. 4. Outer active and reactive power and DC voltage loop 


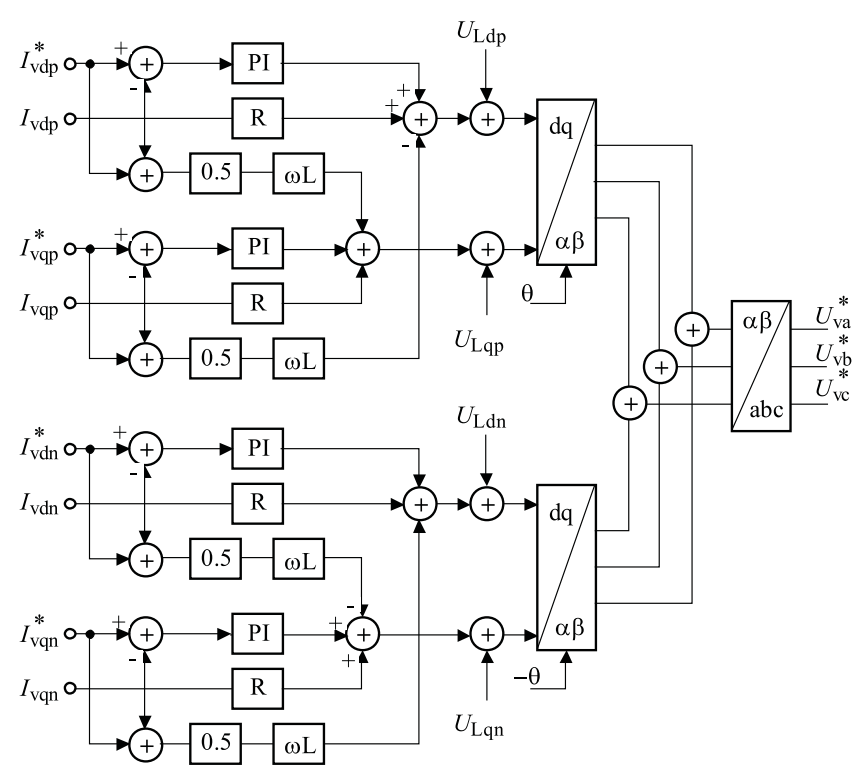

Fig. 5. Inner Current Controller

\subsection{Inner current loop}

For each of the phases we can write (Fig. 2)

$$
U_{L}-u_{v}=L \frac{\mathrm{d} i_{v}}{\mathrm{~d} t}+R i_{v}
$$

During unbalanced operation [8] the expression for the voltage drop over the reactor $(R, L)$ holds for positive as well as for negative-sequence voltages and currents. The voltages drops are described by the differential equation

$$
\begin{gathered}
\frac{\mathrm{d}}{\mathrm{d} t}\left[\begin{array}{c}
i_{v a X} \\
i_{v b X} \\
i_{v c X}
\end{array}\right]=\left[\begin{array}{ccc}
-\frac{R}{L} & 0 & 0 \\
0 & -\frac{R}{L} & 0 \\
0 & 0 & -\frac{R}{L}
\end{array}\right]\left[\begin{array}{l}
i_{v a X} \\
i_{v b X} \\
i_{v c X}
\end{array}\right]- \\
{\left[\begin{array}{ccc}
\frac{1}{L} & 0 & 0 \\
0 & \frac{1}{L} & 0 \\
0 & 0 & \frac{1}{L}
\end{array}\right]\left[\begin{array}{l}
u_{v a X} \\
u_{v b X} \\
u_{v c X}
\end{array}\right]+\left[\begin{array}{ccc}
\frac{1}{L} & 0 & 0 \\
0 & \frac{1}{L} & 0 \\
0 & 0 & \frac{1}{L}
\end{array}\right]\left[\begin{array}{l}
u_{L a X} \\
u_{L b X} \\
u_{L c X}
\end{array}\right] .}
\end{gathered}
$$

Where $X=(p)$ for positive sequence and $(n)$ for negative sequence. Equation (7) can be transformed to the $\alpha \beta$ frame. This gives for the voltages and currents:

$$
\begin{array}{r}
\frac{\mathrm{d}}{\mathrm{d} t}\left[\begin{array}{c}
i_{v \alpha X} \\
i_{v \beta X}
\end{array}\right]=\left[\begin{array}{cc}
-\frac{R}{L} & 0 \\
0 & -\frac{R}{L}
\end{array}\right]\left[\begin{array}{c}
i_{v \alpha X} \\
i_{v \beta X}
\end{array}\right]-\left[\begin{array}{cc}
\frac{1}{L} & 0 \\
0 & \frac{1}{L}
\end{array}\right]\left[\begin{array}{l}
u_{v \alpha X} \\
u_{v \beta X}
\end{array}\right] \\
+\left[\begin{array}{cc}
\frac{1}{L} & 0 \\
0 & \frac{1}{L}
\end{array}\right]\left[\begin{array}{c}
u_{L \alpha X} \\
u_{L \beta X}
\end{array}\right] .
\end{array}
$$

Equation (8) can be further transferred into the rotating $d q$-frame:

$$
\begin{array}{r}
\frac{\mathrm{d}}{\mathrm{d} t}\left[\begin{array}{c}
i_{v d p} \\
i_{v q p}
\end{array}\right]=\left[\begin{array}{cc}
-\frac{R}{L} & \omega \\
\omega & -\frac{R}{L}
\end{array}\right]\left[\begin{array}{c}
i_{v d p} \\
i_{v q p}
\end{array}\right]-\left[\begin{array}{cc}
\frac{1}{L} & 0 \\
0 & \frac{1}{L}
\end{array}\right]\left[\begin{array}{l}
u_{v d p} \\
u_{v q p}
\end{array}\right] \\
+\left[\begin{array}{cc}
\frac{1}{L} & 0 \\
0 & \frac{1}{L}
\end{array}\right]\left[\begin{array}{l}
u_{L d p} \\
u_{L q p}
\end{array}\right] .
\end{array}
$$

and

$$
\begin{array}{r}
\frac{\mathrm{d}}{\mathrm{d} t}\left[\begin{array}{c}
i_{v d n} \\
i_{v q n}
\end{array}\right]=\left[\begin{array}{cc}
-\frac{R}{L} & \omega \\
\omega & -\frac{R}{L}
\end{array}\right]\left[\begin{array}{c}
i_{v d n} \\
i_{v q n}
\end{array}\right]-\left[\begin{array}{cc}
\frac{1}{L} & 0 \\
0 & \frac{1}{L}
\end{array}\right]\left[\begin{array}{l}
u_{v d n} \\
u_{v q n}
\end{array}\right] \\
+\left[\begin{array}{cc}
\frac{1}{L} & 0 \\
0 & \frac{1}{L}
\end{array}\right]\left[\begin{array}{l}
u_{L d n} \\
u_{L q n}
\end{array}\right] .
\end{array}
$$

The positive and negative sequence voltages of the VSC side are obtained from (9) and (10):

$$
\begin{aligned}
& u_{v d p}=u_{L d p}-R i_{v d p}+\omega L i_{v q p}-L \frac{\mathrm{d}}{\mathrm{d} t} i_{v d p}, \\
& u_{v q p}=u_{L q p}-R i_{v q p}-\omega L i_{v d p}-L \frac{\mathrm{d}}{\mathrm{d} t} i_{v q p}
\end{aligned}
$$

and

$$
\begin{aligned}
& u_{v d n}=u_{L d n}-R i_{v d n}-\omega L i_{v q n}-L \frac{\mathrm{d}}{\mathrm{d} t} i_{v d n}, \\
& u_{v q n}=u_{L q n}-R i_{v q n}-\omega L i_{v d n}-L \frac{\mathrm{d}}{\mathrm{d} t} i_{v q n} .
\end{aligned}
$$

The mean voltages over the sample period $k$ to $k+1$ are derived by integrating (11), (12), (13) and (14) from $k T_{s}$ to $(k+1) T_{s}$ and dividing by $T_{s}$ (where $T_{s}$ is the sampling time).

$$
\begin{gathered}
\bar{u}_{v d p}=\bar{u}_{L d p}-R \bar{i}_{v d p}+ \\
\omega L \bar{i}_{v q p}-\frac{L}{T_{s}}\left\{i_{v d p}(k+1)-i_{v d p}(k)\right\}, \\
\bar{u}_{v q p}=\bar{u}_{L q p}-R \bar{i}_{v q p}- \\
\omega L \bar{i}_{v q p}-\frac{L}{T_{s}}\left\{i_{v q p}(k+1)-i_{v q p}(k)\right\}
\end{gathered}
$$

and

$$
\begin{aligned}
\bar{u}_{v d n}= & \bar{u}_{L d n}-R \bar{i}_{v d n}- \\
& \omega L \bar{i}_{v q n}-\frac{L}{T_{s}}\left\{i_{v d n}(k+1)-i_{v d n}(k)\right\}, \\
\bar{u}_{v q n}= & \bar{u}_{L q n}-R \bar{i}_{v q n}+ \\
& \omega L \bar{i}_{v q n}-\frac{L}{T_{s}}\left\{i_{v q n}(k+1)-i_{v q n}(k)\right\},
\end{aligned}
$$

By assuming linear current and constant network voltage (the network voltage varies very little during a switching time period) during one sample period $T_{s}$ we obtain from (15) through (18):

$$
\begin{aligned}
u_{v d p}(k+1)=u_{L d p}(k) & -\frac{R}{2}\left\{i_{v d p}(k+1)+i_{v d p}(k)\right\} \\
+ & \frac{\omega L}{2}\left\{i_{v q p}(k+1)+i_{v q p}(k)\right\} \\
& -\frac{L}{T_{s}}\left\{i_{v d p}(k+1)-i_{v d p}(k)\right\}
\end{aligned}
$$




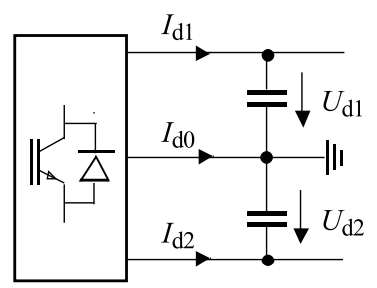

Fig. 6. DC Voltages and Currents of the Three-Level Bridge
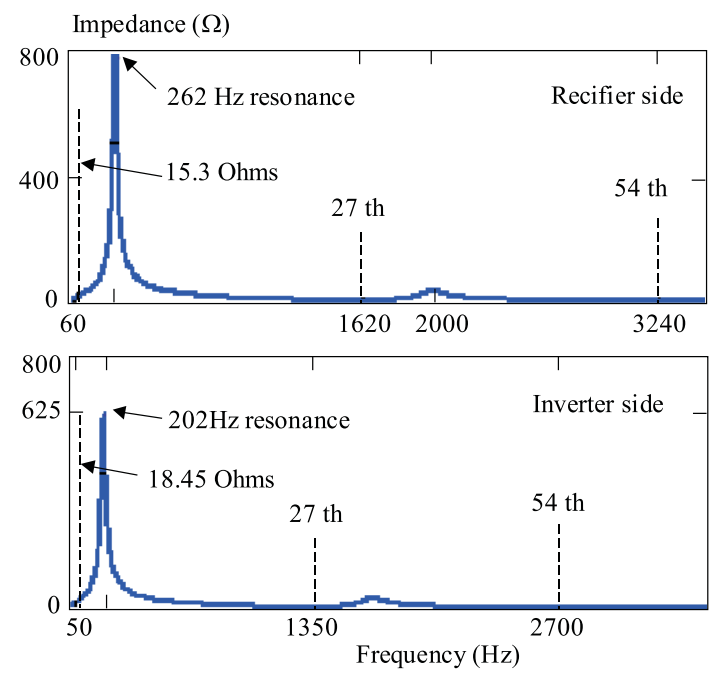

Fig. 7. Positive-Sequence impedances of the two AC networks

$$
\begin{aligned}
u_{v q p}(k+1)=u_{L q p}(k) & -\frac{R}{2}\left\{i_{v q p}(k+1)+i_{v q p}(k)\right\} \\
& -\frac{\omega L}{2}\left\{i_{v d p}(k+1)+i_{v d p}(k)\right\} \\
& -\frac{L}{T_{s}}\left\{i_{v q p}(k+1)-i_{v q p}(k)\right\},
\end{aligned}
$$

$$
\begin{aligned}
u_{v d n}(k+1)=u_{L d n}(k) & -\frac{R}{2}\left\{i_{v d n}(k+1)+i_{v d n}(k)\right\} \\
& -\frac{\omega L}{2}\left\{i_{v q n}(k+1)+i_{v q n}(k)\right\} \\
& -\frac{L}{T_{s}}\left\{i_{v d n}(k+1)-i_{v d n}(k)\right\},
\end{aligned}
$$

$$
\begin{aligned}
u_{v q n}(k+1)=u_{L q n}(k) & -\frac{R}{2}\left\{i_{v q n}(k+1)+i_{v q n}(k)\right\} \\
& -\frac{\omega L}{2}\left\{i_{v d n}(k+1)+i_{v d n}(k)\right\} \\
& -\frac{L}{T_{s}}\left\{i_{v q n}(k+1)-i_{v q n}(k)\right\},
\end{aligned}
$$

The control is based on (19), (20), (21) and (22), where the voltages and currents at time $(k+1)$ are thus equal to the reference values at time step $(k)$.

\subsection{DC voltage balance control}

The difference between the DC side voltages (positive and negative) are controlled to keep the DC side of the three level bridge balanced (ie, equal pole voltages) in steady-state. Small deviations between the pole voltages may occur at changes of active/reactive converter current or due to nonlinearity on lack of precision in the execution of the pulse width modulated bridge voltage. Furthermore, deviations between the pole voltages may be due to inherent unbalance in the circuit components impedance. The DC midpoint current $I_{d 0}$ determines the difference $U_{d 0}$ between the upper and lower DC voltages (Fig. 6).

The DC midpoint current determines the difference between the upper and lower DC voltages, ie

$$
I_{d 0}=-\left(I_{d 1}+I_{d 2}\right)=-2 C \frac{\mathrm{d}}{\mathrm{d} t}\left(U_{d 1}-U_{d 2}\right)=-2 C \frac{\mathrm{d}}{\mathrm{d} t} U_{d 0}
$$

By changing the conduction time of the switches in a pole it is possible to change the average of the DC midpoint current $I_{d 0}$ and thereby control the difference voltage $U_{d 0}$. For example, a positive difference $\left(U_{d 0} \geq 0\right)$ can be decreased to zero if the amplitude of the reference voltage which generates a positive midpoint current is increased at the same time as the amplitude of the reference voltage which generates a negative DC midpoint current is decreased. This is done by the addition of an offset component to the sinusoidal reference voltage. Consequently, the bridge voltage becomes distorted, and to limit the distortion effect, the control has to be slow. Finally, for better performance this function should be activated in the station controlling the DC voltage.

\section{SIMULATIONS RESULTS}

The dynamic performance of the transmission system is verified by simulating the frequency response of the two $\mathrm{AC}$ systems and the following types of disturbances:

1. Steps on the regulators references,

2. Recovery from severe perturbations in the AC system.

\subsection{Frequency response of the AC systems}

Figure 7 demonstrates the magnitude, seen from the busbar where the filter is connected, of the combined filter and $\mathrm{AC}$ network impedance as a function of frequency.

Notice the two minimum impedances on the $Z$ magnitudes of the AC systems, these series resonances are created by the $27^{\text {th }}$ and $54^{\text {th }}$ harmonic filters. They occur at $1620 \mathrm{~Hz}$ and $3240 \mathrm{~Hz}$ on the $60 \mathrm{~Hz}$ system $(1350 \mathrm{~Hz}$ and $2700 \mathrm{~Hz}$ on the $50 \mathrm{~Hz}$ ). The low principal natural frequency, coinciding whit the parallel resonance at $262 \mathrm{~Hz}$ on the rectifier side and $202 \mathrm{~Hz}$ on the inverter side, is a determining factor in the development of overvoltages and interaction with the DC system. 

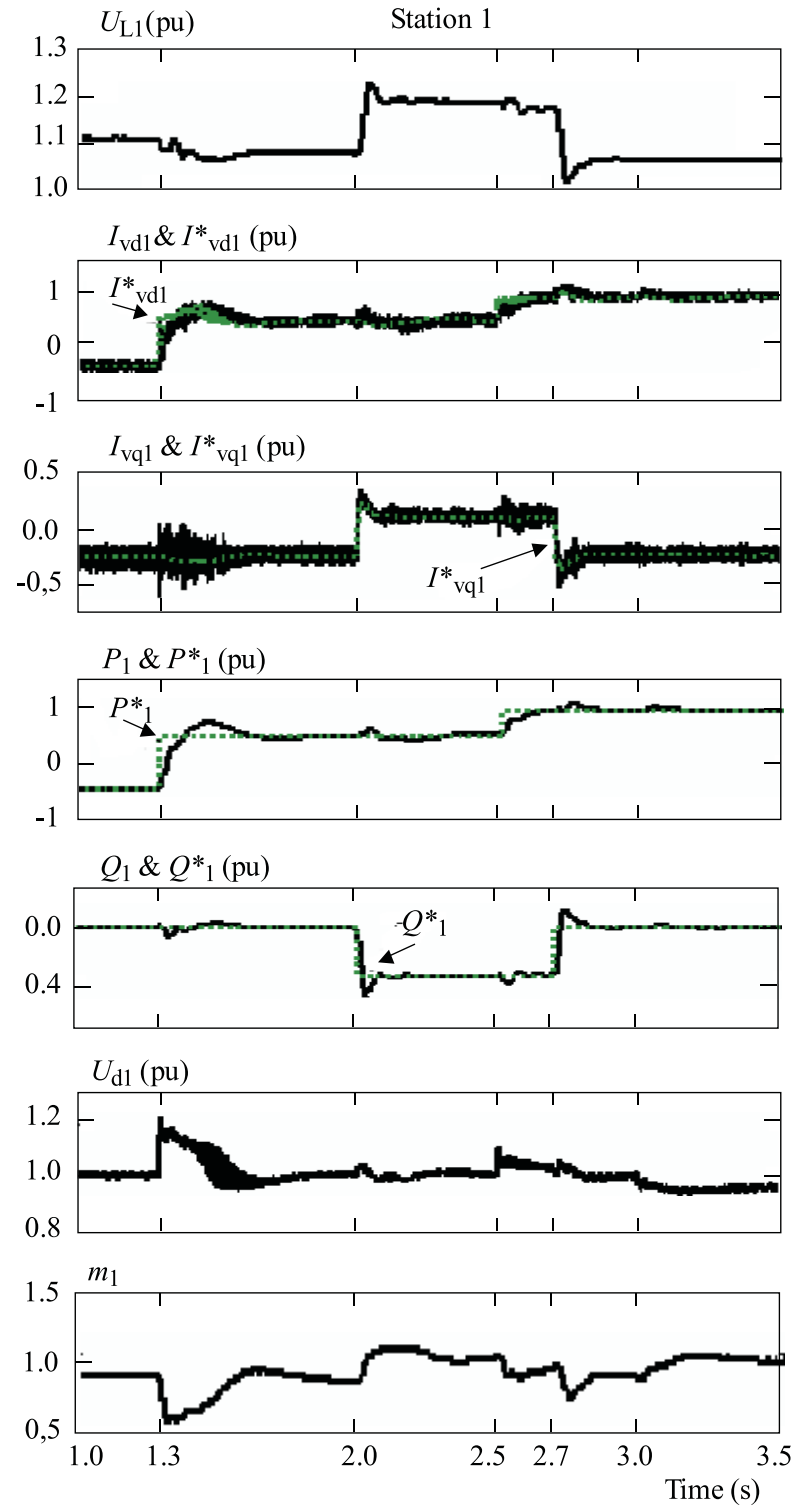
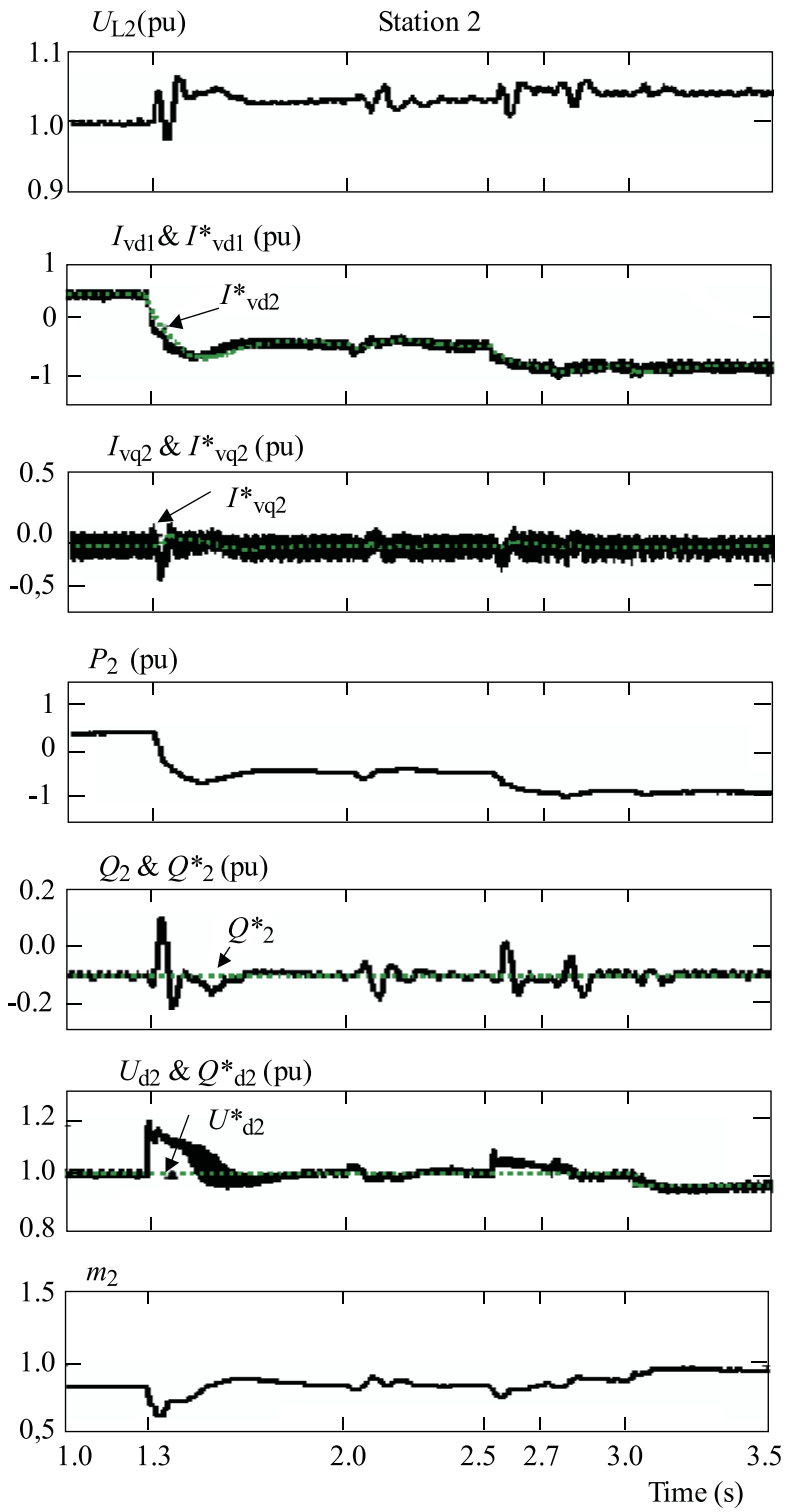

Fig. 8. Steps on the regulators references

\subsection{Step responses}

In order to test the dynamic responses of the VSCHVDC regulators, three test cases have been studied:

- At $t=1.3 \mathrm{~s}$ the reference active power of converter $1\left(P_{1}^{*}\right)$ is changed from $-0.5 \mathrm{pu}$ to $+0.5 \mathrm{pu}$, and at $t=2.5 \mathrm{~s}$ from $+0.5 \mathrm{pu}$ to $+1.0 \mathrm{pu}$

- From $t=2.0 \mathrm{~s}$ to $t=2.7 \mathrm{~s}$ the reference reactive power of converter $1\left(Q_{1}^{*}\right)$ is changed from $0.0 \mathrm{pu}$ to $-0.4 \mathrm{pu}$.

- At $t=3.0 \mathrm{~s}$ the reference DC voltage of converter 2 is changed from $+1.0 \mathrm{pu}$ to $+0.095 \mathrm{pu}$.

First the converter 1 active power reference value is changed from from $-0.5 \mathrm{pu}$ to $+0.5 \mathrm{pu}$ and then set to $1.0 \mathrm{pu}$ at $t=2.5 \mathrm{~s}$. As can be seen from Fig. 8 the active power can track the reference of the active power.

The transferred active powers at both sides change the direction which causes transients on the DC voltage then returns to the reference value due to the DC voltage con- troller. The AC voltages at the filter bus ( $U_{L 1}$ and $\left.U_{L 2}\right)$, can be kept constant except for some transients that occur when both the step changes are applied. The active power flow is adjusted to the new setting within $30 \mathrm{~ms}$. At $t=2.0 \mathrm{~s}$ the reactive power step change is applied, this step change cause transients on the DC voltage, but, as expected, the step change of the active power causes a much higher transient than that with the change in reactive power. At $t=2.5 \mathrm{~s}$ the active power change to $1 \mathrm{pu}$, and later another step is also applied on the reference DC voltage of the inverter at $t=3.0 \mathrm{~s}$. The reactive power and the DC voltage can track the references of the reactive power and the $\mathrm{DC}$ voltage. The active and reactive power responses are decoupled by the control design.

\subsection{AC side perturbations}

A remote three phase to ground fault was first applied at $t=1.5 \mathrm{~s}$ during $0.12 \mathrm{~s}$ ( 5 cycles on $60 \mathrm{~Hz}$ system $)$ at 

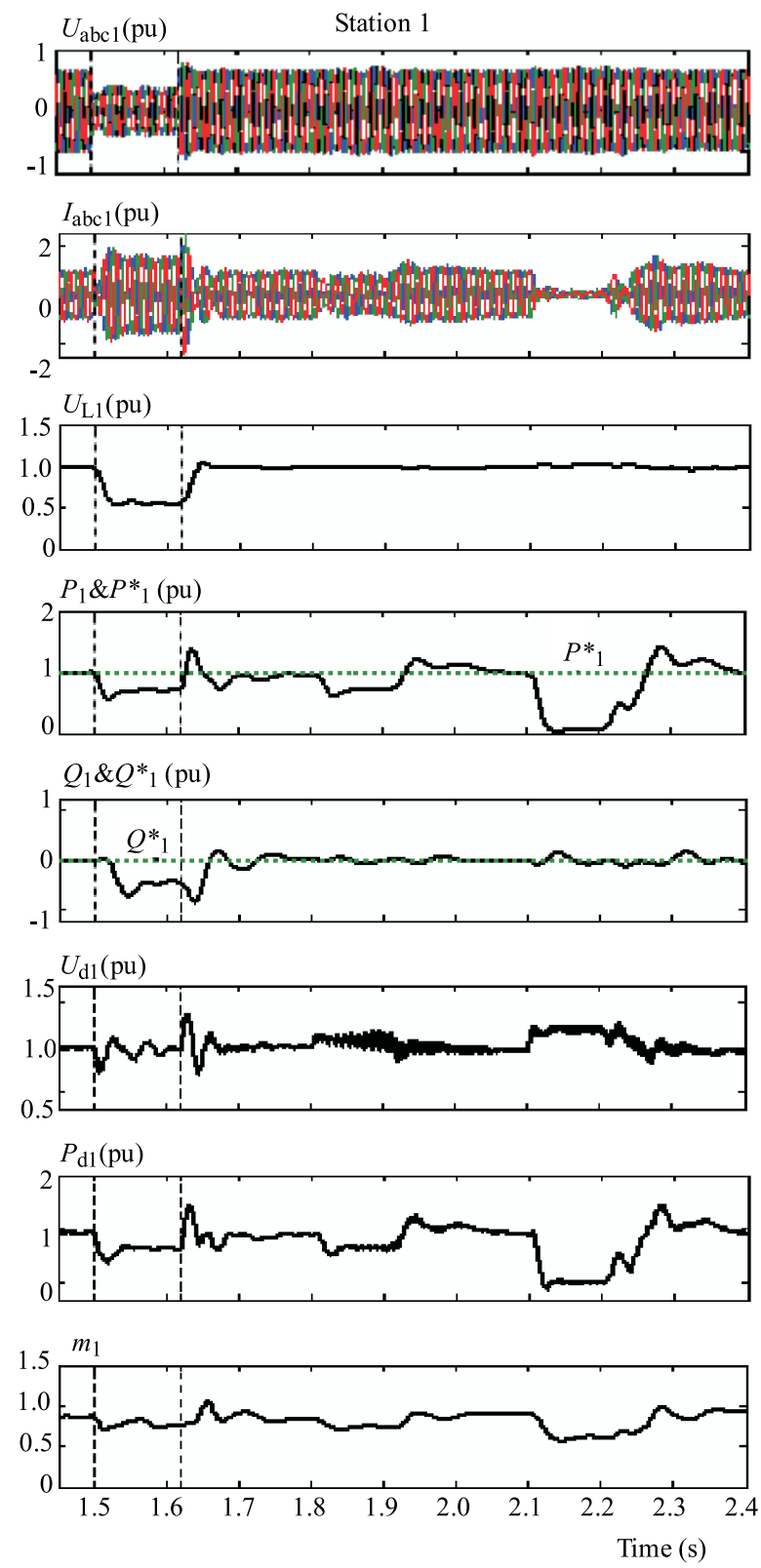
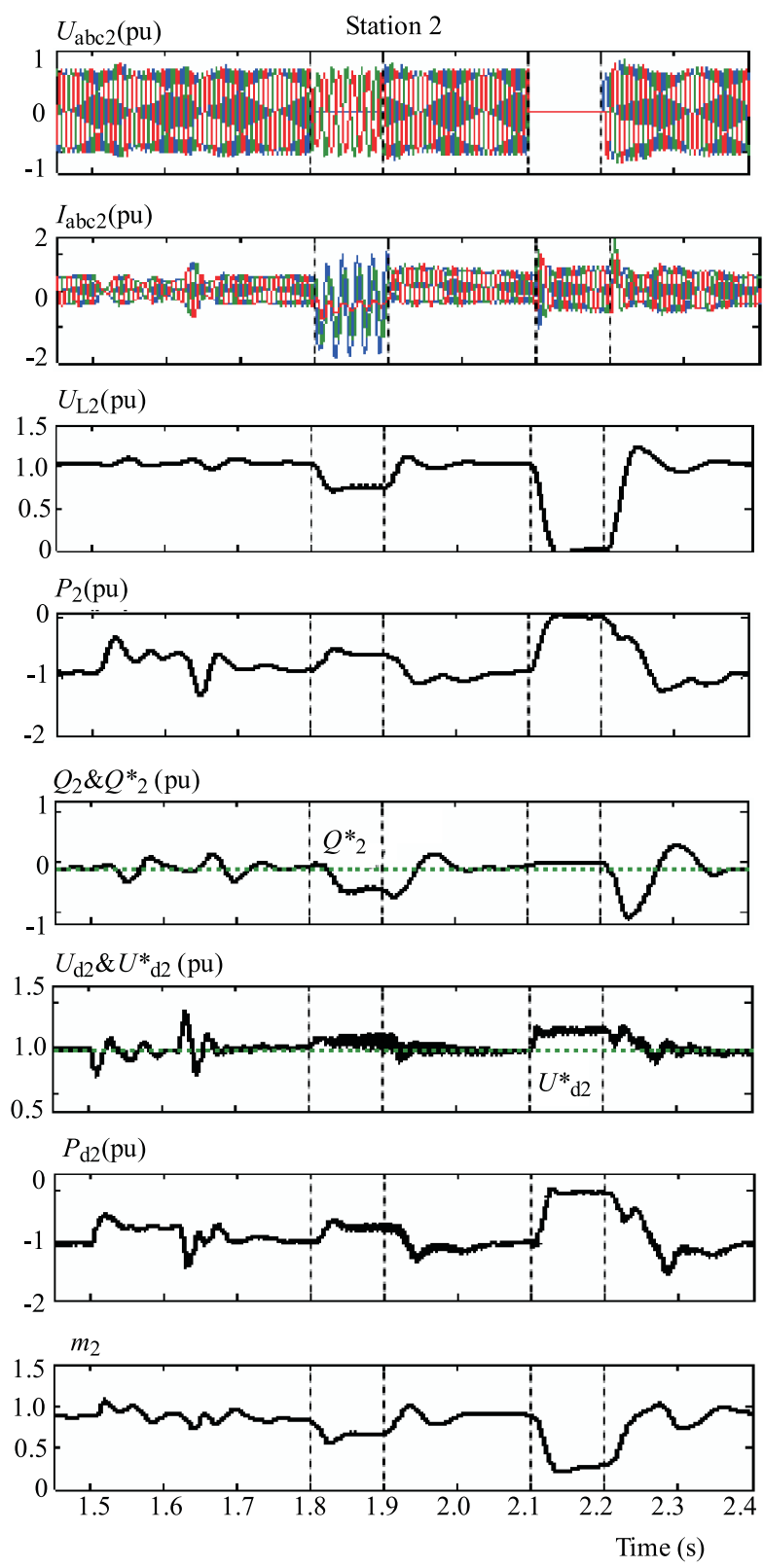

Fig. 9. AC side perturbations

station 1. A second perturbation follows. A single phase to ground fault was applied at $t=1.8 \mathrm{~s}$ during $0.10 \mathrm{~s}$ ( 5 cycles on $50 \mathrm{~Hz}$ system) at station 2 in order to investigate the behavior of VSC-HVDC during unbalanced faults. Then, a three-phase to ground fault is applied at station 2 at $t=2.1 \mathrm{~s}$ and is cleared at 5 cycles after the fault, $i e$, at $t=2.2 \mathrm{~s}$. Figure 9 presents the simulations results. From the simulation, it can be noted that during the two first faults $(t=1.5 \mathrm{~s}$ at station 1 and $t=1.8 \mathrm{~s}$ at station 2 ), the active power flow is $1.0 \mathrm{pu}$, transmitted from converter 1 to converter 2, and is kept constant during the fault. The DC voltage drops and it contains an oscillation during the faults.

Consequently the transferred DC power contains also the oscillation. During these two faults the transmitted power can be kept constant except a small oscillation dur- ing the fault. All oscillations in voltages and currents at both systems, means that the phase voltages and currents at both systems are unbalanced. During the severe three-phase fault at station 2 at $t=2.1 \mathrm{~s}$, the AC voltage at station 2 side is decreased to $0.1 \mathrm{pu}$ during the fault and recovers fast and successfully to $1.0 \mathrm{pu}$ voltage after clearing the fault. The transmitted power flow is reduced to very low value during the fault and recovers to $1.0 \mathrm{pu}$ after the fault. The DC voltage, which can be controlled to $1.0 \mathrm{pu}$ during the fault, has some oscillations at the beginning of the fault and at clearing the fault, and its maximum transient value is about $1.2 \mathrm{pu}$. On the other hand, the phase currents at station 1 side decrease to low values to reduce the power flow. 


\section{CONCLUSION}

In this paper, we have presented the steady-state and dynamic performances of an asynchronous back-to-back HVDC link based on voltage source converters, during step changes of the active and reactive powers, balanced and unbalanced faults. In all cases the proposed control strategy has been shown to provide fast and satisfactory dynamic responses of the proposed system. Besides controlling the through power flow, it can supply reactive power and provide independent dynamic voltage control at its two terminals. The two converters can be paralleled to double the reactive power capability supplied to one side or the other. Higher voltage designs can be used with transmission lines or cables to form point-to- point or multi-terminal transmission links. More sophisticated controls can be used to provide additional network benefits. From the simulation, it can be obtained that the VSC-HVDC can fulfil fast and bi-directional power transfers. It can be obtained also that during a single-phase fault the transmitted power can be kept constant except a small oscillation during the fault. However, during a three-phase fault; the decreased voltage at the converter terminals strongly reduces the power flow by the DC link. When the fault is cleared, normal operation is recovered fast.

\section{APPENDIX}

Data for the system model

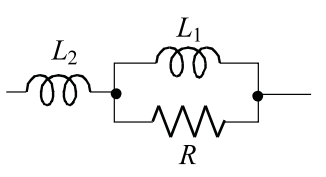

\section{Station 1:}

The AC system 1 representing a strong system (2000 MVA, $S C R=10)$, consists of one source with an equivalent impedance of: $R=13.79 \Omega, L_{1}=31.02 \mathrm{mH}, L_{2}=$ $62.23 \mathrm{mH}$.

\section{Station 2:}

The AC system 2 representing a strong system (1000 MVA, $S C R=5)$, consists of one source with an equivalent impedance of: $R=27.58 \Omega, L_{1}=62.04 \mathrm{mH}, L_{2}=$ $124.46 \mathrm{mH}$.

\section{REFERENCES}

[1] SMITH, D.: Cross Sound HVDC Link Extends IGBT Power, Modern Power Systems - Transmission \& Distribution, October 2000, pp. 31-36.
[2] WEIMERS, L.: New Markets Need New Nechnology, International Conference on Power System Technology, PowerCon 2000, vol. 2, 2000, pp. 873-877.

[3] SCHETTLER, F.-HUANG, H.-CHRISTL, N. : HVDC Transmission Systems using Voltage Sourced Converters Design and Applications, IEEEPower Engineering Society Summer Meeting, vol. 2, 2000, pp. 715-720.

[4] WEIMERS, L.: HVDC Light: A New Technology for a Better Environment, IEEE Power Engineering Review 18 No. 8 (August 1998), 19-20.

[5] ASPLUND, G.: Application of HVDC Light to Power System Enhancement, IEEE Power Engineering Society Winter Meeting, vol. 4, 2000, pp. 2498-2503.

[6] GOLE, A. M.: HVDC Course Notes, Manitoba HVDC Research Centre, Canada, 2000.

[7] LINDBERG, A.-LARSSON, T. : PWM and Control of Three Level Voltage Source Converters in an Hvdc Back-to-back Station, Sixth International Conference on $\mathrm{AC}$ and $\mathrm{DC}$ Power Transmission, 29 April -3 May 1996, pp. 297-302.

[8] LIE XU-ANDERSEN, B. R.-CARTWRIGHT, P.: Control of VSC Transmission Systems under Unbalanced Network Conditions, Transmission and Distribution Conference and Exposition, 2003 IEEE PES, vol. 2, 7-12 September 2003, pp. 626-632.

Received 23 April 2009

Khatir Mohamed was born in Ain Temouchent, Algeria, in 1977. He received the Eng. degree in electro technical engineering, and the Master degree from the Djillali Liabes University of Sidi Bel-Abbes (Algeria), in 2002 and 2006 respectively. He is now a PhD Candidate in the Electrical Engineering Department of Djillali Liabes University. His main field of interest includes HVDC and FACTS.

Zidi Sid-Ahmed was born in Sidi Bel Abbes, Algeria. He received the diploma of Electro technical Engineering degree from the university of Science and Technology of Oran, Algeria, the Master degree from the University of Djillali Liabes of Sidi Bel-Abbes, Algeria in 1994, the PhD degree from the University of Sidi Bel Abbes, Algeria, in 2005. He is currently interested in the HVDC link, FACTS and transient in power systems.

Hadjeri Samir received the Master degree in Electrical Engineering from the University of Laval, Quebec, Canada, in 1990, the PhD degree from the University of Sidi Bel-Abbes, Algeria, in 2003. Since 1991 he has been a teaching member at the Department of Electrical Engineering of Djillali Liabes University. His research is focused on HVDC, FACTS and power system analysis.

Fellah Mohammed-Karim was born in Oran, Algeria, in 1963. He received the Eng. degree in Electrical Engineering from the University of Sciences and Technology, Oran, Algeria, in 1986, and the PhD degree from the National Polytechnic Institute of Lorraine (Nancy, France) in 1991. Since 1992, he has been Professor at the University of Sidi Bel Abbes (Algeria) and Director of the Intelligent Control and Electrical Power Systems Laboratory at this University. His current research interest includes power electronics, HVDC links, and drives. 